\title{
The Limits of Vinylidene Fluoride RAFT
}

\section{Polymerization.}

Marc Guerre, ${ }^{a}$ S. M. Wahidur Rahaman, ${ }^{b}$ Bruno Améduri, ${ }^{a}$ Rinaldo Poli, ${ }^{b, c^{*}}$ Vincent Ladmiral ${ }^{a}$ *

${ }^{a}$ Institut Charles Gerhardt, Ingénierie et Architectures Macromoléculaires, UMR 5253 CNRS, UM, ENSCM, Place Eugène Bataillon, UM, 34095 Montpellier Cedex 5 France.

${ }^{\mathrm{b}}$ CNRS, LCC (Laboratoire de Chimie de Coordination), Université de Toulouse, UPS, INPT, 205

Route de Narbonne, BP 44099, F-31077 Toulouse Cedex 4, France.

${ }^{c}$ Institut Universitaire de France, 1, rue Descartes, 75231 Paris Cedex 05, France.

SUPPORTING INFORMATION 
(S1) (\%) $-\mathrm{CF}_{2}-\mathrm{CH}_{2}-\mathrm{XA}$

$=\frac{\frac{1}{2} \int_{4.02}^{4.17}-\mathrm{CF}_{2}-\mathrm{CH}_{2}-\mathrm{XA}}{\frac{1}{3} \int_{1.71}^{1.87}-\mathrm{CF}_{2}-\mathrm{CH}_{3}+\frac{1}{2} \int_{3.26}^{3.52}-\mathrm{CH}_{2}-\mathrm{CF}_{2}-\mathrm{XA}+\int_{6.01}^{6.48}-\mathrm{CH}_{2}-\mathrm{CF}_{2} \mathrm{H}+\frac{1}{2} \int_{4.02}^{4.17}-\mathrm{CF}_{2}-\mathrm{CH}_{2}-\mathrm{XA}}$

(S2) (\%) $-\mathrm{CH}_{2}-\mathrm{CF}_{2}-\mathrm{XA}$

$=\frac{\frac{1}{2} \int_{3.26}^{3.52}-\mathrm{CH}_{2}-\mathrm{CF}_{2}-\mathrm{XA}}{\frac{1}{3} \int_{1.71}^{1.87}-\mathrm{CF}_{2}-\mathrm{CH}_{3}+\frac{1}{2} \int_{3.26}^{3.52}-\mathrm{CH}_{2}-\mathrm{CF}_{2}-\mathrm{XA}+\int_{6.01}^{6.48}-\mathrm{CH}_{2}-\mathrm{CF}_{2} \mathrm{H}+\frac{1}{2} \int_{4.02}^{4.17}-\mathrm{CF}_{2}-\mathrm{CH}_{2}-\mathrm{XA}}$

(S3) (\%) $-\mathrm{CH}_{2}-\mathrm{CF}_{2} \mathrm{H}$

$=\frac{\int_{6.01}^{6.48}-\mathrm{CH}_{2}-\mathrm{CF}_{2} \mathrm{H}}{\frac{1}{3} \int_{1.71}^{1.87}-\mathrm{CF}_{2}-\mathrm{CH}_{3}+\frac{1}{2} \int_{3.26}^{3.52}-\mathrm{CH}_{2}-\mathrm{CF}_{2}-\mathrm{XA}+\int_{6.01}^{6.48}-\mathrm{CH}_{2}-\mathrm{CF}_{2} \mathrm{H}+\frac{1}{2} \int_{4.02}^{4.17}-\mathrm{CF}_{2}-\mathrm{CH}_{2}-\mathrm{XA}}$

(S4) $(\%)-\mathrm{CF}_{2}-\mathrm{CH}_{3}$

$$
=\frac{\frac{1}{3} \int_{1.71}^{1.87}-\mathrm{CF}_{2}-\mathrm{CH}_{3}}{\frac{1}{3} \int_{1.71}^{1.87}-\mathrm{CF}_{2}-\mathrm{CH}_{3}+\frac{1}{2} \int_{3.26}^{3.52}-\mathrm{CH}_{2}-\mathrm{CF}_{2}-\mathrm{XA}+\int_{6.01}^{6.48}-\mathrm{CH}_{2}-\mathrm{CF}_{2} \mathrm{H}+\frac{1}{2} \int_{4.02}^{4.17}-\mathrm{CF}_{2}-\mathrm{CH}_{2}-\mathrm{XA}}
$$

(S5) $(\%) R-\mathrm{CH}_{2}-\mathrm{CF}_{2}-=\frac{\frac{1}{3} \int_{1.19}^{1.25} R-P V D F}{\frac{1}{3} \int_{1.19}^{1.25} R-P V D F+\frac{1}{2} \int_{4.30}^{4.39} D M C-P V D F}$

(S6) (\%) DMC $-\mathrm{CH}_{2}-\mathrm{CF}_{2}-=\frac{\frac{1}{2} \int_{4.30}^{4.39} D M C-P V D F}{\frac{1}{3} \int_{1.19}^{1.25} R-P V D F-+\frac{1}{2} \int_{4.30}^{4.39} D M C-P V D F}$

Equations used to calculate the proportions of chain-ends and to construct Figure 4 (the evolution of the proportion of intrachain and total HH VDF additions $v s$ conversion for three RAFT polymerizations) were calculated using the equations described in our earlier report. ${ }^{1}$ 


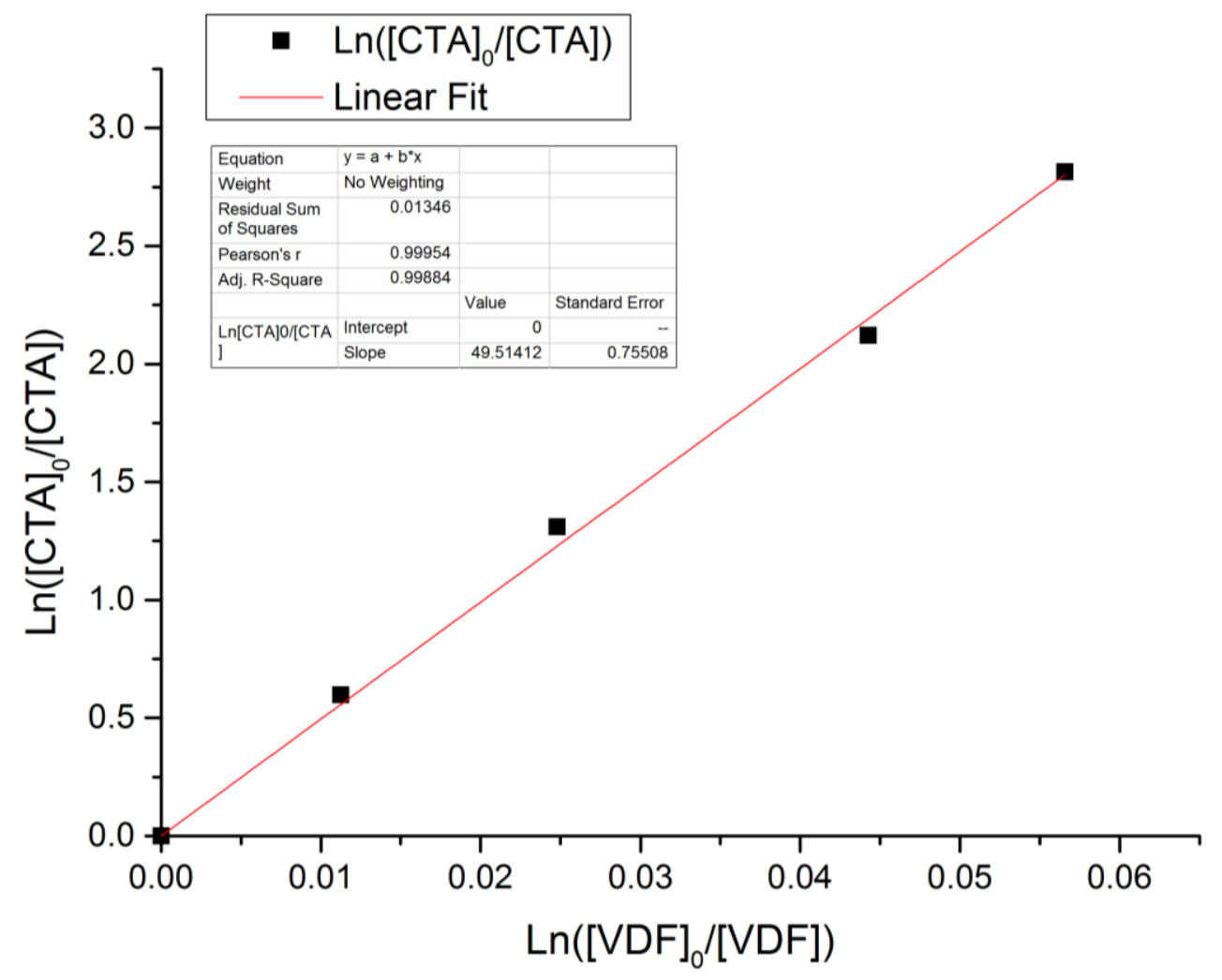

Figure S1. Determination of the transfer constant of $O$-ethyl-S-(1methoxycarbonyl)ethyldithiocarbonate CTA with VDF using O'Brien and Gornick's method. ${ }^{2}$

Figures S1 displays the plots of $\operatorname{Ln}\left([\mathrm{CTA}]_{0} /[\mathrm{CTA}]\right)$ versus $\mathrm{Ln}\left([\mathrm{VDF}]_{0} /[\mathrm{VDF}]\right)$. The slope of the

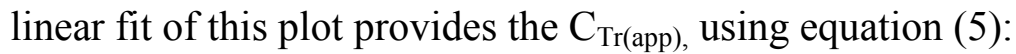

(5) $\operatorname{Ln}\left(\frac{[C T A]_{0}}{[C T A]}\right)=C_{T r(a p p)} \operatorname{Ln}\left(\frac{[V D F]_{0}}{[V D F]}\right)$

$\mathrm{C}_{\operatorname{Tr}(\text { app })}=49$ at $73{ }^{\circ} \mathrm{C}$ 


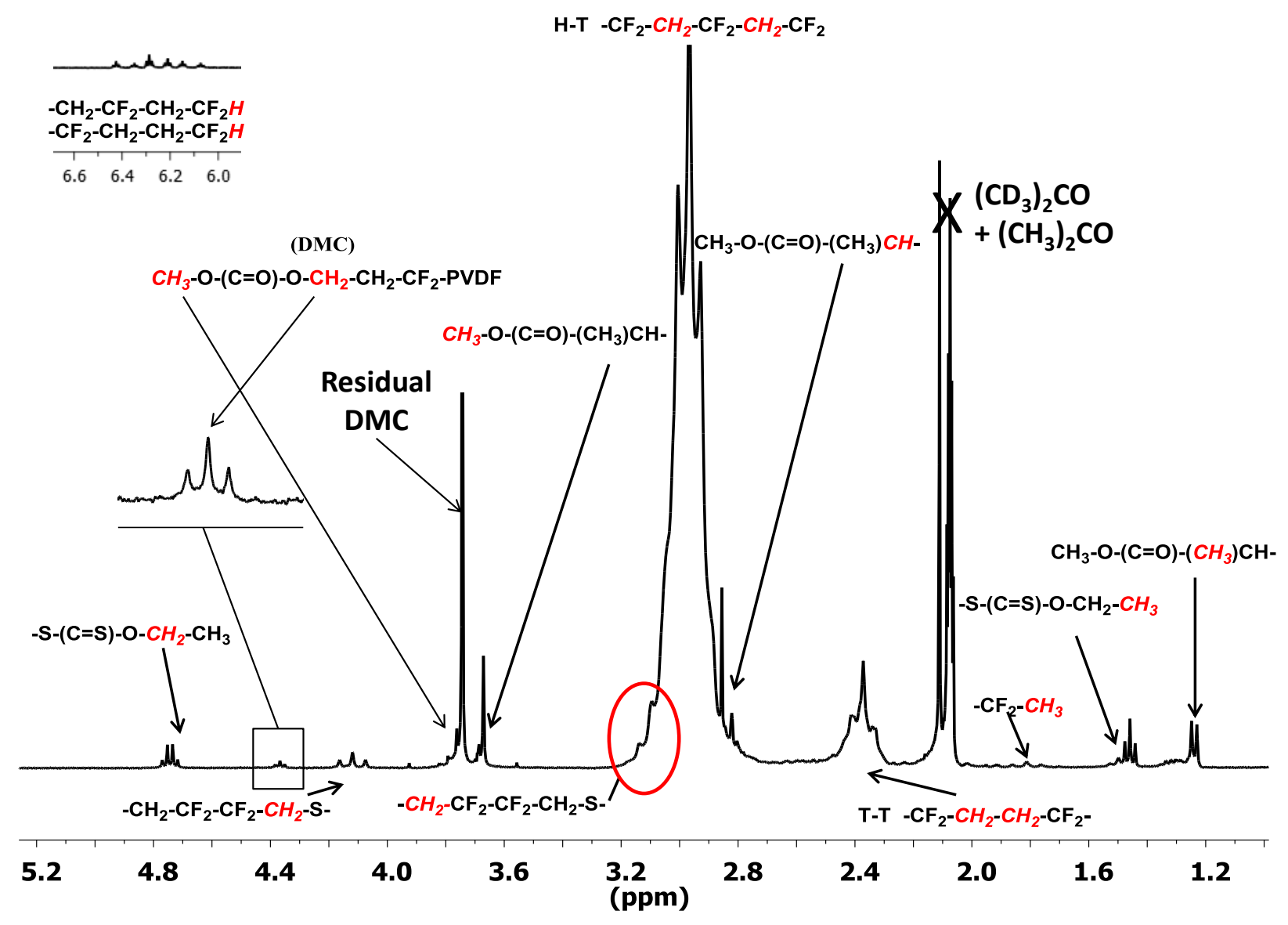

Figure S2. Expansion of the $1.0-5.2 \mathrm{ppm}$ region of the ${ }^{1} \mathrm{H} \mathrm{NMR}$ spectrum in $\left(\mathrm{CD}_{3}\right)_{2} \mathrm{CO}$ of PVDF homopolymer (run 13, Table 1) synthesized via RAFT polymerization. 


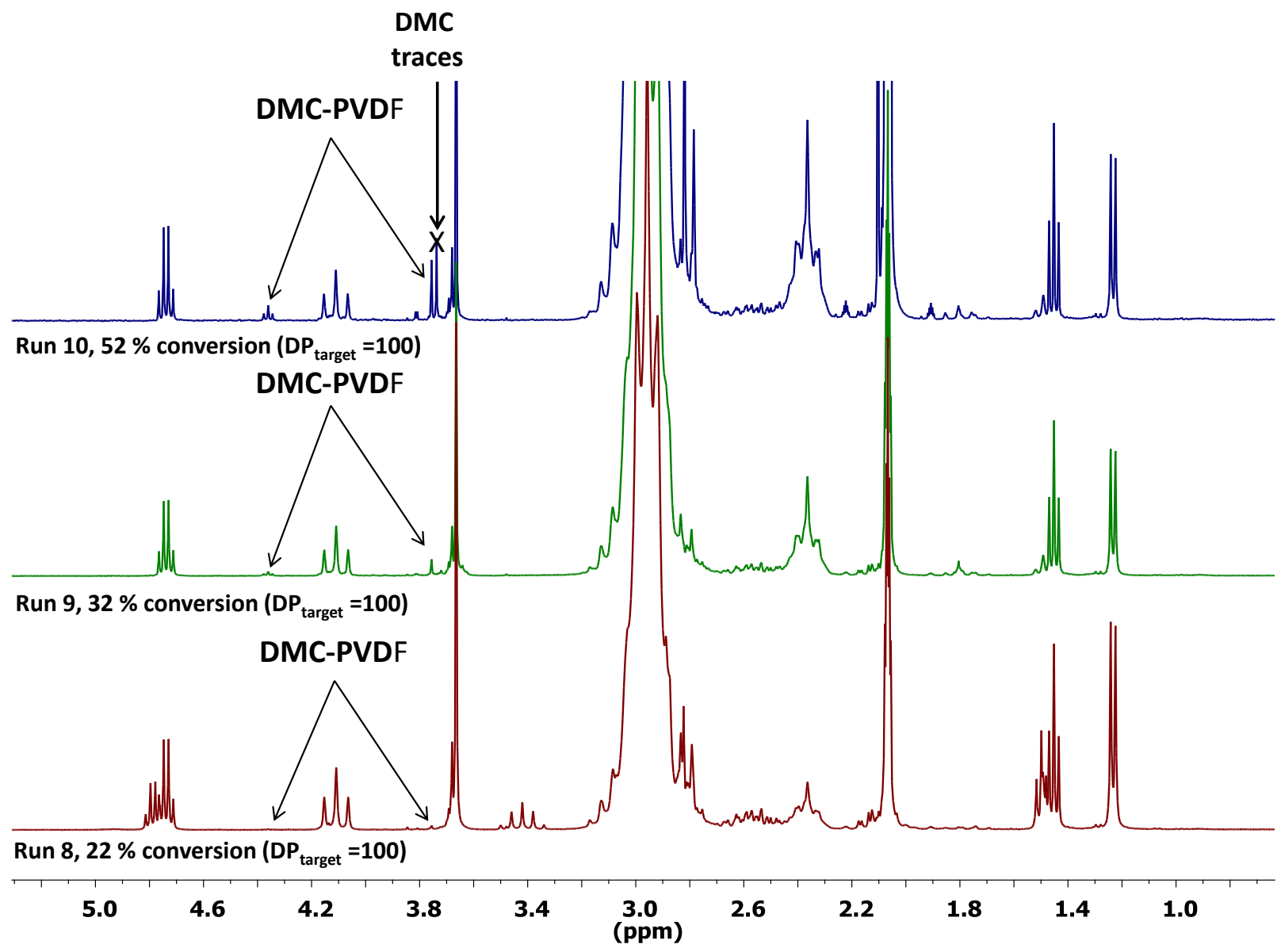

Figure S3. Evolution of the PVDF chains initiated by DMC radicals (DMC-PVDF) during the course of a VDF RAFT polymerization $\left(\mathrm{DP}_{\text {target }}=100\right)$. The DMC moiety at the $\alpha$-chain-end is characterized by two ${ }^{1} \mathrm{H}$ NMR signals at 3.73 and $4.34 \mathrm{ppm}$. The ${ }^{1} \mathrm{H}$ NMR spectra were recorded in $\left(\mathrm{CD}_{3}\right)_{2} \mathrm{CO}$. 


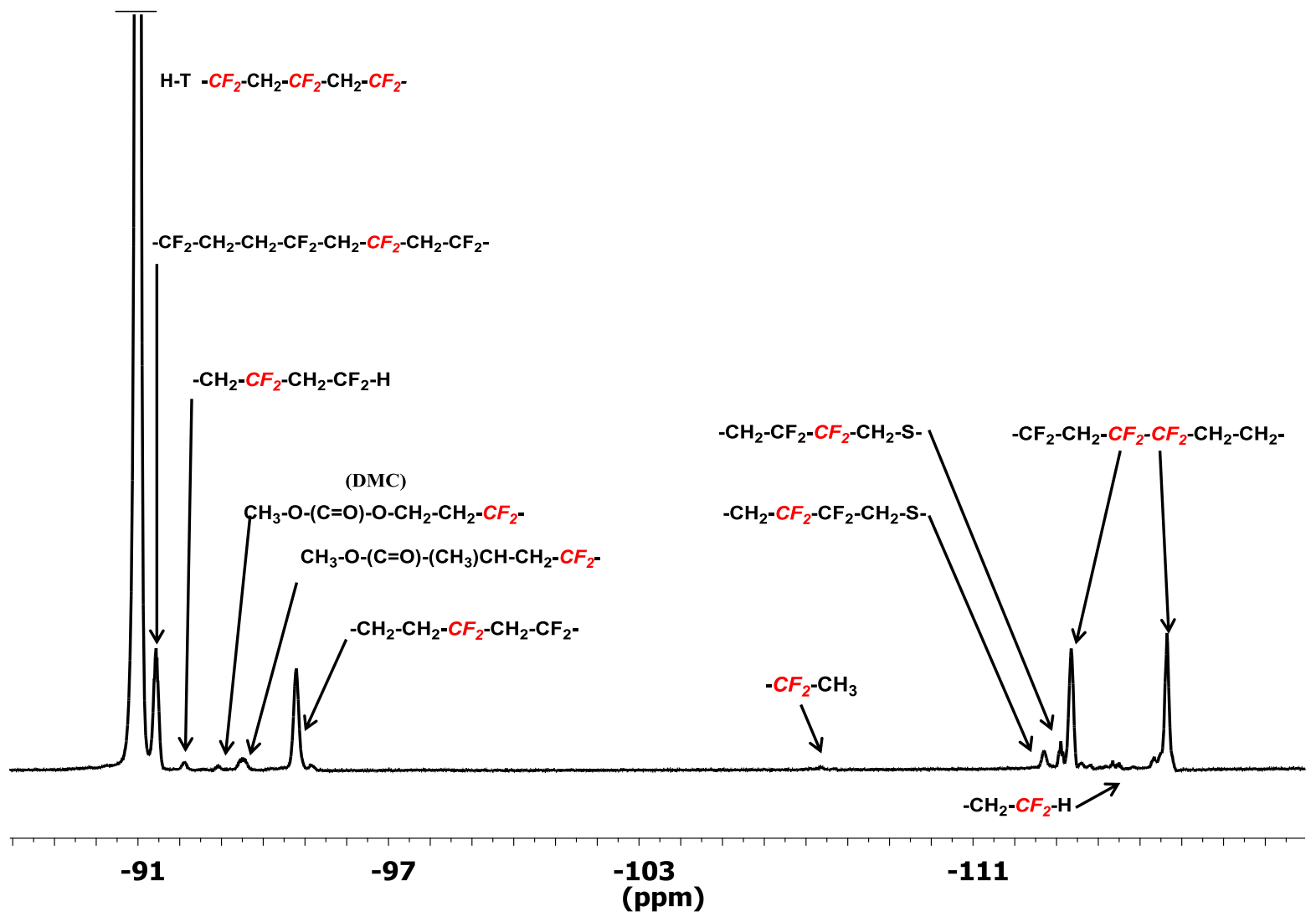

Figure S4. Expansion of the -88 to $-119 \mathrm{ppm}$ region of the ${ }^{19} \mathrm{~F}$ NMR spectrum in $\left(\mathrm{CD}_{3}\right)_{2} \mathrm{CO}$ of PVDF homopolymer (run 13, Table 1) synthesized via RAFT polymerization. 
Table S1. Energies, enthalpies, Gibbs energies of all optimized geometries.

\begin{tabular}{|c|c|c|c|c|}
\hline Compound & $E$ (hartrees) & \begin{tabular}{|l}
$\mathrm{H}_{298.15}$ \\
(hartrees)
\end{tabular} & $\begin{array}{l}\mid \begin{array}{l}\mathrm{G}_{298.15} \\
\text { (hartrees) }\end{array} \\
\end{array}$ & $\begin{array}{l}\mathrm{G}_{343.15} \\
\text { (hartrees) }^{\mathrm{a}}\end{array}$ \\
\hline \multicolumn{5}{|l|}{ (a) Radicals } \\
\hline $\mathrm{CHF}_{2} \mathrm{CH}_{2}^{-}$ & -277.3819182 & -277.330618 & $\mid-277.363927$ & -277.3689544 \\
\hline $\mathrm{CH}_{3} \mathrm{CF}_{2}{ }^{-}$ & -277.3942109 & -277.341757 & -277.374487 & -277.379427 \\
\hline$(\mathrm{MeO}) \mathrm{C}(\mathrm{S}) \mathrm{S}^{\circ}$ & -949.1387931 & -949.081962 & -949.119578 & -949.1252554 \\
\hline \multicolumn{5}{|l|}{ (b) Monomers } \\
\hline $\mathrm{CH}_{2}=\mathrm{CF}_{2}$ & -276.8179418 & -276.77658 & $\mid-276.807351$ & -276.8119953 \\
\hline \multicolumn{5}{|l|}{ (c) Chain transfer agents } \\
\hline $\mathrm{CHF}_{2} \mathrm{CH}_{2}-\mathrm{SC}(\mathrm{S})(\mathrm{OMe})$ & -1226.623079 & -1226.509289 & -1226.558343 & -1226.565747 \\
\hline $\mathrm{CH}_{3} \mathrm{CF}_{2}-\mathrm{SC}(\mathrm{S})(\mathrm{OMe})$ & -1226.623112 & -1226.510636 & -1226.559231 & -1226.566565 \\
\hline \multicolumn{5}{|l|}{ (d) VDF propagation } \\
\hline \multicolumn{5}{|l|}{ d1. Head to tail } \\
\hline $\mathrm{CH}_{3} \mathrm{CF}_{2} \cdot \mathrm{CH}_{2} \mathrm{CF}_{2} \cdot \mathrm{TS}$ & -554.2069944 & -554.112686 & -554.159811 & -554.1669236 \\
\hline $\mathrm{CH}_{3} \mathrm{CF}_{2}-\mathrm{CH}_{2} \mathrm{CF}_{2}^{\circ}$ & -554.2596083 & -554.162115 & -554.206273 & -554.2129378 \\
\hline \multicolumn{5}{|l|}{ d2. Head to head } \\
\hline $\mathrm{CH}_{3} \mathrm{CF}_{2} \mathrm{CF}_{2} \mathrm{CH}_{2} \cdot \mathrm{TS}$ & -554.204510 & -554.110865 & -554.156331 & -554.1631932 \\
\hline $\mathrm{CH}_{3} \mathrm{CF}_{2}-\mathrm{CF}_{2} \mathrm{CH}_{2}^{\circ}$ & -554.2563001 & -554.161183 & -554.204725 & -554.2112968 \\
\hline \multicolumn{5}{|l|}{ d3. Tail to tail } \\
\hline $\mathrm{CHF}_{2} \mathrm{CH}_{2}{ }^{\prime \cdots} \mathrm{CH}_{2} \mathrm{CF}_{2} \cdot \mathrm{TS}$ & -554.1966673 & -554.102442 & -554.148653 & -554.1556277 \\
\hline $\mathrm{CHF}_{2} \mathrm{CH}_{2}-\mathrm{CH}_{2} \mathrm{CF}_{2}^{-}$ & -554.2540946 & -554.155206 & -554.19951 & -554.2061968 \\
\hline \multicolumn{5}{|l|}{ d4. Tail to head } \\
\hline $\mathrm{CHF}_{2} \mathrm{CH}_{2}{ }^{\prime \prime} \mathrm{CF}_{2} \mathrm{CH}_{2}{ }^{\circ} \mathrm{TS}$ & -554.190701 & -554.09724 & -554.142244 & -554.1490365 \\
\hline $\mathrm{CHF}_{2} \mathrm{CH}_{2}-\mathrm{CF}_{2} \mathrm{CH}_{2}^{-}$ & -554.249403 & -554.15306 & -554.197573 & -554.2042914 \\
\hline \multicolumn{5}{|c|}{ (e) MacroCTA degenerative and non-degenerative radical exchange } \\
\hline \multicolumn{5}{|l|}{ e1. Head-head (degenerative) } \\
\hline $\mathrm{CH}_{3} \mathrm{CF}_{2}-\mathrm{SC}(\mathrm{OMe}) \mathrm{S} \cdots \cdot \mathrm{CF}_{2} \mathrm{CH}_{3} \mathrm{TS}$ & -1504.017061 & -1503.851318 & $\mid-1503.916319$ & -1503.92613 \\
\hline $\mathrm{CH}_{3} \mathrm{CF}_{2}-\mathrm{SC}^{*}(\mathrm{OMe}) \mathrm{S}-\mathrm{CF}_{2} \mathrm{CH}_{3}$ & -1504.033495 & -1503.866567 & -1503.929195 & -1503.938647 \\
\hline
\end{tabular}




\begin{tabular}{|l|l|l|l|l|}
\hline $\mathrm{CH}_{3} \mathrm{CF}_{2}-\mathrm{SC}(\mathrm{OMe}) \mathrm{S}^{\circ} \mathrm{CH}_{2} \mathrm{CHF}_{2} \mathrm{TS}$ & -1504.006925 & -1503.841794 & -1503.905873 & -1503.915544 \\
\hline $\mathrm{CH}_{3} \mathrm{CF}_{2}-\mathrm{SC}(\mathrm{OMe}) \mathrm{S}-\mathrm{CH}_{2} \mathrm{CHF}_{2}$ & -1504.028759 & -1503.860603 & -1503.924011 & -1503.933581 \\
\hline $\mathrm{CH}_{3} \mathrm{CF}_{2}{ }^{\circ} \mathrm{SC}(\mathrm{OMe}) \mathrm{S}-\mathrm{CH}_{2} \mathrm{CHF}_{2} \mathrm{TS}$ & -1504.013737 & -1503.846844 & -1503.911662 & -1503.921445 \\
\hline e3. Tail-tail (degenerative) & -1504.007386 & -1503.840705 & -1503.904122 & -1503.913694 \\
\hline $\mathrm{CHF}_{2} \mathrm{CH}_{2}-\mathrm{SC}(\mathrm{OMe}) \mathrm{S}^{\circ}{ }^{\circ} \mathrm{CH}_{2} \mathrm{CHF}_{2} \mathrm{TS}$ & -1504.023796 & -1503.854494 & -1503.918067 & -1503.927662 \\
\hline $\mathrm{CHF}_{2} \mathrm{CH}_{2}-\mathrm{SC}(\mathrm{OMe}) \mathrm{S}-\mathrm{CH}_{2} \mathrm{CHF}_{2}$ & & & \\
\hline
\end{tabular}

${ }^{\mathrm{a}}$ Extrapolation done on the basis $\mathrm{G}_{343.15} \approx \mathrm{H}_{298.15}-343.15 \cdot \mathrm{S}_{298.15}$.

Table S2. Cartesian coordinates and views of all optimized geometries.

(a) Radicals
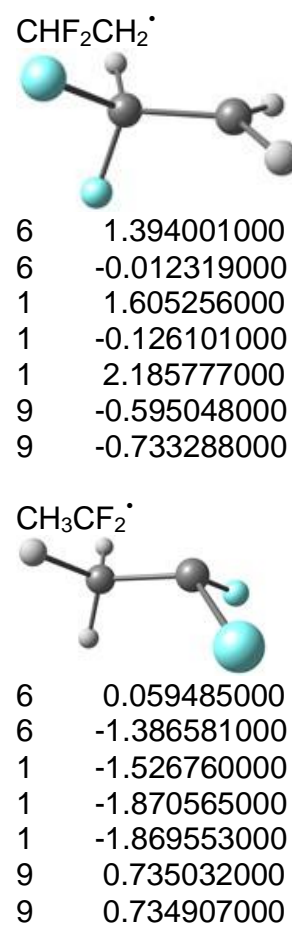

(MeO)C(S)S

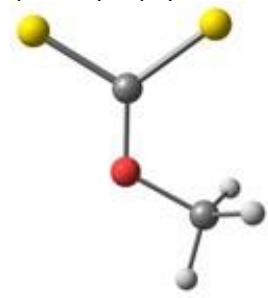

$16 \quad 1.736278000$

$\begin{array}{ll}6 & 0.122262000\end{array}$

$8-0.840806000$

$\begin{array}{ll}16 & -0.059249000\end{array}$

$6-2.203329000$
$-0.107134000$ 0.007573000

$-0.523019000$

0.065360000

0.377679000

1.137805000

$-1.062544000$

0.000229000

0.000075000

0.000221000

0.890101000

$-0.890889000$

1.098602000

$-1.098742000$
$-0.085236000$

0.354372000

$-1.065566000$

1.446414000

0.476297000

$-0.178214000$

$-0.096448000$

$-0.305567000$

0.044979000

1.137790000

$-0.366469000$

$-0.365766000$

0.064360000

0.064303000

$\begin{array}{ll}-0.629578000 & -0.000015000\end{array}$

$\begin{array}{ll}-0.147474000 & 0.000058000\end{array}$

$\begin{array}{ll}-1.050048000 & 0.000022000\end{array}$

$1.524074000-0.000003000$

$-0.590138000$

$-0.000024000$
$1-2.405410000$

$1-2.405500000$

$1-2.808709000$

0.006032000

0.005896000

$-1.497813000$

(b) Monomers

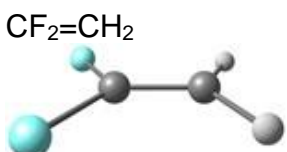

$6-1.386768000$

$6-0.062812000$

$9 \quad 0.699065000$

90.695002000

$1-1.924716000$

$-1.924408000$

0.002088000

0.000473000

1.082140000

$-1.084340000$

$-0.937027000$

0.941462000

$-0.896070000$

0.896092000

$-0.000123000$

0.000517000

$-0.000250000$

0.000097000

$-0.000050000$

$-0.000253000$

$-0.001776000$

(c) Chain transfer agents

$\mathrm{CHF}_{2} \mathrm{CH}_{2}-\mathrm{SC}(\mathrm{S})(\mathrm{OMe})$

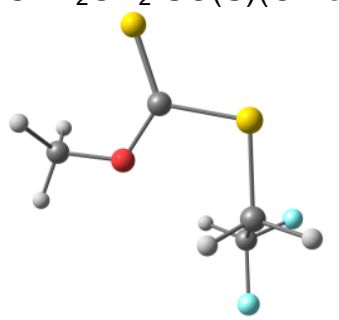

$6-1.388504000$

$\begin{array}{lll}6 & -2.139901000 & 0.290759000\end{array}$

$\begin{array}{lll}1 & -1.514938000 & 0.912329000\end{array}$

$\begin{array}{lll}16 & 0.039507000 & -1.312514000\end{array}$

$6 \quad 1.315328000$

$-1.312514000$

0.907451000

1.115151000

$\begin{array}{ll}16 & 2.818127000\end{array}$

$-0.673688000$

1.871772000

2.153075000

2.256150000

2.082942000

2.705447000

2.065589000

1.325110000

3.085697000

$-1.061251000$

0.538475000

$-2.065970000$

$-0.928721000$

0.878223000

$-0.311861000$

$-0.965455000$

0.424652000

0.073669000

0.186556000

$-0.340214000$

$-0.054321000$

$-1.076646000$

0.649297000

0.097855000

1.533052000

1.436919000 
1.090199000

$-0.205844000$

$-0.182032000$

$-0.260780000$

$-1.432006000$

$-2.521939000$

$-1.720436000$

2.431724000

3.213755000

1.136308000

1.062776000

$-0.000102000$

$6 \quad-1.739920000$

0.093570000 $-3.070870000$ $-3.858173000$

$-0.619464000$

0.141307000

$-3.174583000$

$-3.174924000$

$-1.241038000$

$-1.240682000$

0.894919000

$-1.641560000$

$-1.642260000$

0.895670000

$16-0.382514000$

$6 \quad 1.124096000$

$-1.168765000$

$-0.245886000$

0.933171000

1.065294000

$-1.052479000$

1.914417000

2.090846000

1.728319000

2.696436000

1.728387000

2.696846000

2.930133000

\section{(d) VDF propagation}

d1. Head to tail

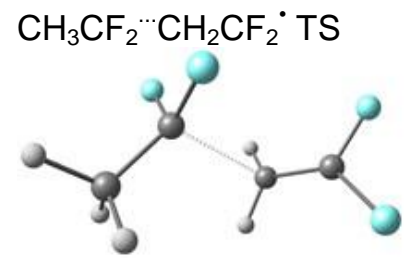

$6 \quad-2.158405000$

1.207936000

$-1.574520000$

2.050013000

$6-1.358932000$

$6 \quad 0.657535000$

1.677277000

$-2.427143000$

$-3.084703000$

$-0.940210000$

$-1.975705000$

0.406299000

0.369504000

2.188369000

2.217070000

$-0.046126000$

0.082305000

0.049552000

1.404482000

1.128328000

$-0.333394000$

$-1.126313000$

1.030245000

$-0.838078000$

$-1.055216000$

1.121924000

\section{$\mathrm{CH}_{3} \mathrm{CF}_{2}-\mathrm{CH}_{2} \mathrm{CF}_{2}{ }^{-}$}

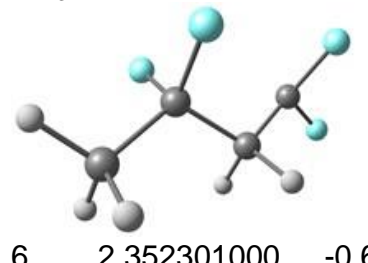

$6 \quad 2.352301000$

$-0.619137000$

$1 \quad 2.359051000$
$-0.000207000$

$-0.000444000$

$-0.893661000$

0.893459000

$-1.093022000$

1.092355000

0.000561000

0.000132000

0.000332000

$-0.000251000$

0.000030000

$-0.892552000$

0.892371000

0.000106000

0.047492000

$-0.335116000$

$-0.045633000$

1.153753000

0.277680000

1.089606000

$-0.542083000$

$-1.291850000$

0.475005000

1.614796000

1.647436000

$-0.240560000$

$-0.284193000$

\section{d2. Head to head}

\section{$\mathrm{CH}_{3} \mathrm{CF}_{2} \cdot \mathrm{CF}_{2} \mathrm{CH}_{2} \cdot \mathrm{TS}$}

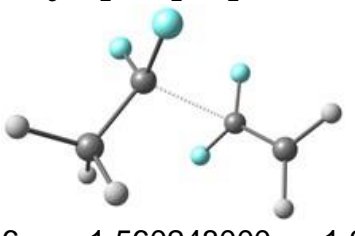

$$
\begin{array}{ll}
6 & 1.560248000 \\
1 & 1.232692000 \\
6 & 1.013457000
\end{array}
$$

$-1.255145000$

$-1.581038000$

$-1.364521000$

$-1.287949000$

$-1.654650000$

$-1.618625000$

1.201817000

2.659521000

1.324937000

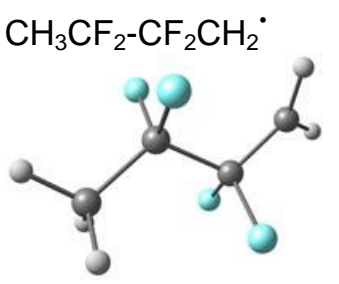

$6 \quad-1.546077000$

$1-1.319949000$

$-0.739621000$

0.790337000

1.573209000

$-1.303983000$

$-2.608819000$

$-1.039052000$

$-1.052605000$

1.079842000

1.122616000

1.200517000

2.567926000

d3. Tail to tail

$\mathrm{CHF}_{2} \mathrm{CH}_{2}{ }^{\cdots} \mathrm{CH}_{2} \mathrm{CF}_{2} \cdot \mathrm{TS}$

1.287506000

2.040159000

$-0.043277000$

0.055866000

0.767576000

$-1.269951000$

0.582123000

0.266309000

1.848336000

1.562044000

1.262548000

$-0.436538000$

$-1.029570000$

$-0.330611000$

0.391370000

0.052733000

0.015317000

1.116467000

$-0.048813000$

$-1.213663000$

2.072941000

1.062336000

$-1.325580000$

$-0.342228000$

1.296438000

$-0.809771000$

$-1.146382000$

0.048530000

$-1.683404000$

0.972568000

0.122913000

$-0.017998000$

$-0.076506000$

0.006120000

1.175666000

$-0.030580000$

$-1.782413000$

$-0.806343000$

$-0.889717000$

0.023648000

0.813291000

$-1.159569000$

1.037376000

0.939394000

1.147868000

$-0.798540000$

$-1.051994000$

0.472528000

1.158640000

$-0.461960000$ 


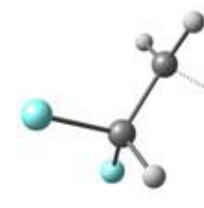

9

$9-1.825636000$

$6 \quad-1.627059000$

$1-1.068415000$

$6-0.953191000$

$1-0.549421000$

$1-1.352531000$

$6 \quad 1.054238000$

$1 \quad 1.254540000$

$1 \quad 0.438363000$

$6 \quad 1.886274000$

$9 \quad 2.780549000$

$9 \quad 1.822095000$

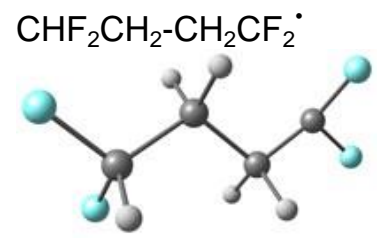

$9 \quad-2.862340000$

$9-2.167248000$

$6-1.886139000$

$1-1.941927000$

$6-0.545296000$

$1-0.360260000$

$1-0.616610000$

$6 \quad 0.587436000$

$1 \quad 0.409619000$

$1 \quad 0.632993000$

$6 \quad 1.917319000$

$9 \quad 2.929997000$

$9 \quad 2.259175000$

0.853176000

$-1.242727000$

0.009726000

$-0.030221000$

0.468713000

1.482832000

0.527766000

$-0.468354000$

$-1.476077000$

$-0.556416000$

$-0.005094000$

$-0.848195000$

1.240210000

d4. Tail to head

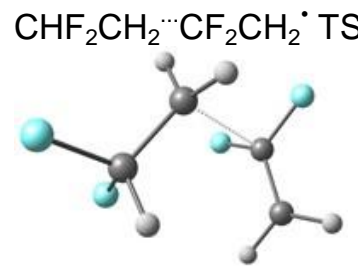

$9-2.795715000$

$9-1.435116000$

$6-1.550438000$

$1-1.527002000$

$6-0.491674000$

$1-0.311338000$

$1-0.388650000$

$6 \quad 1.514295000$

$6 \quad 1.418863000$

$9 \quad 2.197708000$

$9 \quad 1.395091000$

$1 \quad 1.666271000$
0.551306000

$-1.164521000$

0.060687000

$-0.087853000$

0.974100000

1.877259000

1.057178000

$-0.075097000$

$-0.611269000$

1.039993000

$-0.798675000$

$-0.007044000$
$-0.808372000$

0.828245000

$-0.277944000$

$-1.019233000$

0.109315000

$-0.686980000$

0.980438000

1.069772000

1.524110000

1.593110000

0.108770000

$-0.512652000$

$-0.446213000$

$-0.116868000$

$-0.148715000$

0.326858000

1.425270000

$-0.194117000$

0.178402000

$-1.287184000$

0.219272000

$-0.171379000$

1.320292000

$-0.276451000$

$-0.038490000$

0.090876000

$-0.005202000$

$-0.280439000$

0.319078000

1.407860000

$-0.170238000$

0.407400000

$-1.250392000$

0.074862000

1.314945000

$-0.173371000$

$-1.030880000$

2.178717000

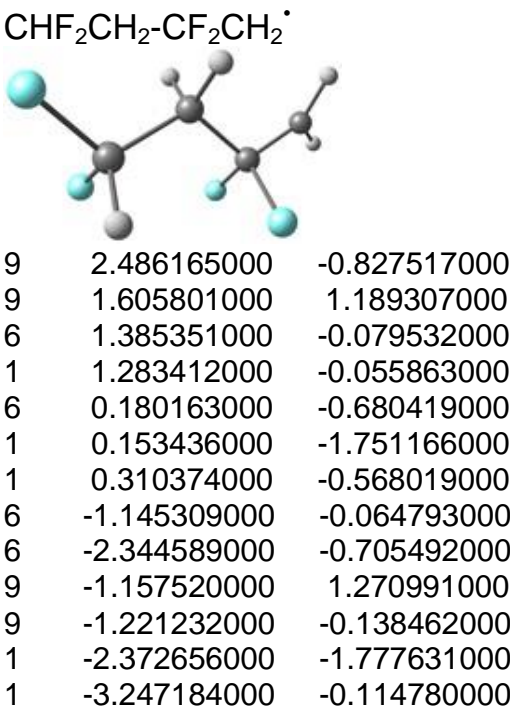

$-0.068382000$

$-0.191796000$

0.247794000

1.339907000

$-0.448223000$

$-0.217443000$

$-1.530292000$

$-0.026238000$

$-0.616745000$

$-0.326758000$

1.362722000

$-0.780691000$

$-0.733091000$

(e) MacroCTA degenerative and nondegenerative radical exchange

e1. Head-head (degenerative)

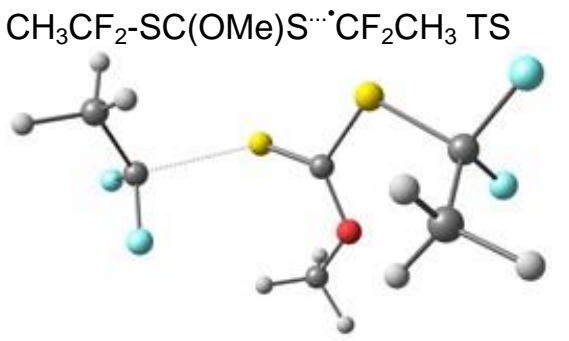

$6 \quad-2.807691000 \quad-0.392322000$

$\begin{array}{lll}-2.568589000 & -0.426759000 & 1.715561000\end{array}$

$\begin{array}{llll}16 & -1.345727000 & -0.801172000 & -0.844229000\end{array}$

$\begin{array}{llll}6 & -0.184783000 & 0.507732000 & -0.613656000\end{array}$

$\begin{array}{llll}8 & -0.612813000 & 1.475967000 & 0.204955000\end{array}$

$\begin{array}{llll}6 & 0.283122000 & 2.565379000 & 0.470123000\end{array}$

$\begin{array}{llll}1 & 0.534414000 & 3.087101000 & -0.458969000\end{array}$

$\begin{array}{llll}1 & 1.199835000 & 2.193688000 & 0.938812000\end{array}$

$\begin{array}{llll}16 & 1.280671000 & 0.421883000 & -1.369011000\end{array}$

$\begin{array}{llll}6 & 3.042866000 & -0.510122000 & 0.325948000\end{array}$

$\begin{array}{llll}6 & 2.982271000 & -1.994919000 & 0.350186000\end{array}$

$\begin{array}{llll}1 & -1.833633000 & 0.330185000 & 1.995035000\end{array}$

$\begin{array}{llll}1 & -2.210837000 & -1.415330000 & 2.013441000\end{array}$

$\begin{array}{llll}1 & -3.518070000 & -0.216943000 & 2.220367000\end{array}$

$\begin{array}{llll}1 & 3.209792000 & -2.387940000 & -0.644091000\end{array}$

$\begin{array}{llll}1 & 1.978033000 & -2.319304000 & 0.635385000\end{array}$

$\begin{array}{llll}1 & 3.707736000 & -2.405592000 & 1.070095000\end{array}$

$\begin{array}{llll}9 & 2.697524000 & 0.085944000 & 1.482297000\end{array}$

$\begin{array}{llll}9 & 4.196593000 & 0.004827000 & -0.116315000\end{array}$

$\begin{array}{llll}9 & -3.343651000 & 0.796117000 & -0.138434000\end{array}$

$\begin{array}{llll}9 & -3.699470000 & -1.367115000 & -0.117744000\end{array}$

$\begin{array}{llll}1 & -0.266016000 & 3.223150000 & 1.146812000\end{array}$

$\mathrm{CH}_{3} \mathrm{CF}_{2}-\mathrm{SC}(\mathrm{OMe}) \mathrm{S}-\mathrm{CF}_{2} \mathrm{CH}_{3}$ 


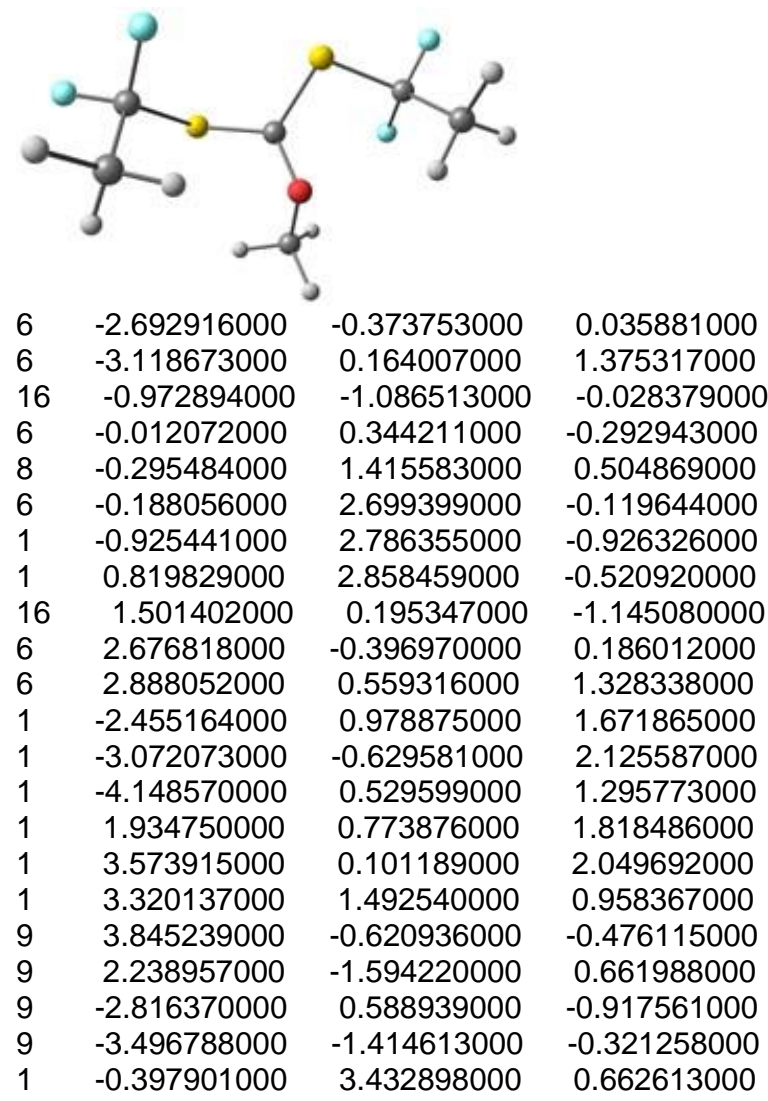

e2. Head-tail (non-degenerative)

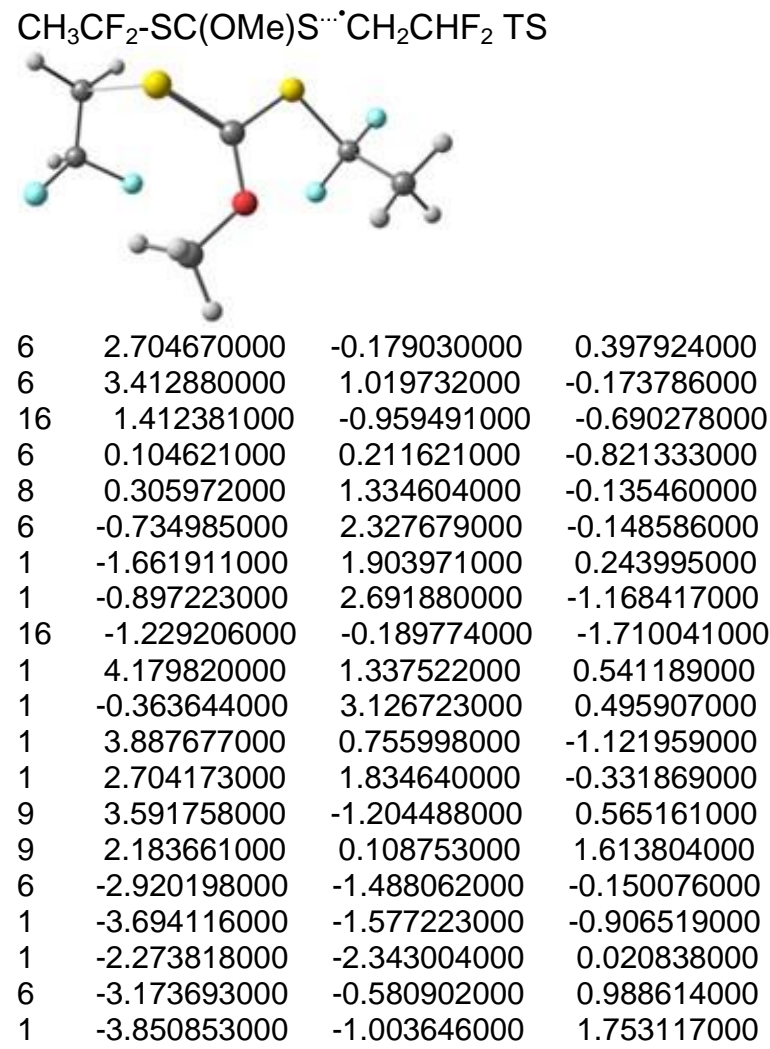

$\begin{array}{rrrr}9 & -3.745881000 & 0.594316000 & 0.566319000 \\ 9 & -2.003810000 & -0.263213000 & 1.633156000\end{array}$

$\mathrm{CH}_{3} \mathrm{CF}_{2}-\mathrm{SC}^{\circ}(\mathrm{OMe}) \mathrm{S}-\mathrm{CH}_{2} \mathrm{CHF}_{2}$

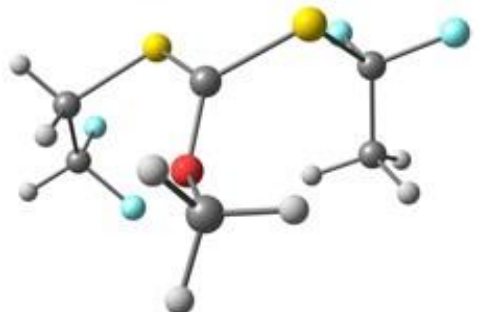

$\begin{array}{lccc}6 & -2.561743000 & 0.338966000 & -0.815491000 \\ 6 & -3.255134000 & -0.289894000 & 0.375786000 \\ 16 & -0.929742000 & -0.403548000 & -1.159936000 \\ 6 & 0.166306000 & 0.853132000 & -0.610863000 \\ 8 & -0.296715000 & 1.635587000 & 0.408132000 \\ 6 & 0.327705000 & 2.910722000 & 0.554966000 \\ 1 & 0.227065000 & 3.502230000 & -0.364330000 \\ 1 & 1.392076000 & 2.803930000 & 0.796091000 \\ 9 & -2.500237000 & -0.169379000 & 1.500397000 \\ 9 & -3.462195000 & -1.618650000 & 0.147318000 \\ 1 & -2.425659000 & 1.409417000 & -0.640166000 \\ 1 & -3.186764000 & 0.195194000 & -1.703354000 \\ 16 & 1.863004000 & 0.600136000 & -0.911426000 \\ 6 & 2.354201000 & -0.768076000 & 0.276327000 \\ 6 & 1.956280000 & -0.545927000 & 1.710050000 \\ 1 & 2.435834000 & 0.356348000 & 2.097786000 \\ 1 & 2.280326000 & -1.410962000 & 2.299251000 \\ 9 & 3.709958000 & -0.842270000 & 0.152167000 \\ 9 & 1.846794000 & -1.949844000 & -0.165418000 \\ 1 & 0.870818000 & -0.442297000 & 1.790889000 \\ 1 & -4.232992000 & 0.179201000 & 0.567229000 \\ 1 & -0.193747000 & 3.404566000 & 1.378523000\end{array}$

$\mathrm{CH}_{3} \mathrm{CF}_{2} \cdot \cdots \mathrm{SC}(\mathrm{OMe}) \mathrm{S}-\mathrm{CH}_{2} \mathrm{CHF}_{2} \mathrm{TS}$

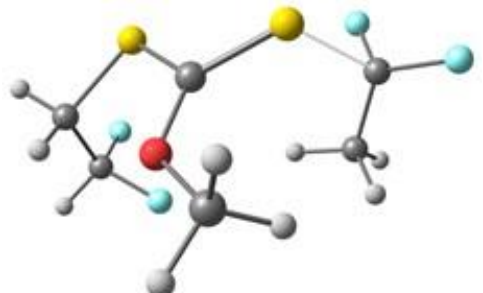

$\begin{array}{lccc}6 & -2.798208000 & 0.149650000 & -0.470829000 \\ 6 & -2.842776000 & -0.971204000 & 0.548816000 \\ 16 & -1.247097000 & 0.193748000 & -1.430182000 \\ 6 & -0.174239000 & 1.199786000 & -0.454341000 \\ 8 & -0.803847000 & 1.838415000 & 0.545190000 \\ 6 & -0.028268000 & 2.715517000 & 1.366292000 \\ 1 & 0.420675000 & 3.511182000 & 0.761734000 \\ 1 & 0.769217000 & 2.164169000 & 1.875700000 \\ 9 & -1.813525000 & -0.858782000 & 1.438387000 \\ 9 & -2.731442000 & -2.179177000 & -0.067584000 \\ 1 & -2.944558000 & 1.113461000 & 0.023837000 \\ 1 & -3.604697000 & -0.000763000 & -1.196710000 \\ 16 & 1.435472000 & 1.255906000 & -0.813511000 \\ 6 & 2.588557000 & -0.898725000 & 0.117475000 \\ 6 & 1.880131000 & -1.285626000 & 1.366163000 \\ 1 & 2.024005000 & -0.510760000 & 2.123764000\end{array}$


1.172353000

e3. Tail-tail (degenerative)

$\mathrm{CHF}_{2} \mathrm{CH}_{2}-\mathrm{SC}(\mathrm{OMe}) \mathrm{S}{ }^{\circ} \mathrm{CH}_{2} \mathrm{CHF}_{2} \mathrm{TS}$

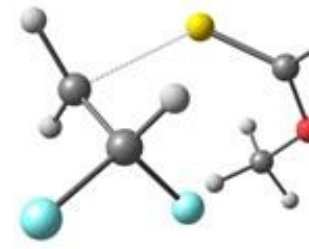

\section{$6 \quad 2.392511000$}

$6 \quad 2.314008000$

$16 \quad 0.924558000$

$6-0.288597000$

$8 \quad 0.216653000$

$6-0.693297000$

$1 \quad-1.238670000$

$1 \quad-1.397947000$

$16-1.875171000$

$6-2.932832000$

$6-1.757377000$

$1 \quad 1.428289000$

$1 \quad-1.050217000$

$1 \quad 2.535962000$

$1 \quad 3.243946000$

$1-3.561877000$

$1 \quad-3.407412000$

$9 \quad 2.305140000$

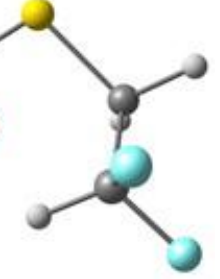

$-0.611062000$

0.757616000

$-0.995779000$

$-1.428782000$

$-1.582915000$

$-1.864745000$

$-2.793475000$

$-1.036412000$

$-1.566746000$

0.787730000

1.656006000

0.892368000

1.667904000

$-1.383921000$

$-0.623671000$

0.579662000

0.816338000

1.724853000
$-0.402674000$

0.248399000

$-1.411168000$

$-0.211594000$

1.015679000

2.089799000

1.894945000

2.203590000

$-0.658039000$

$-0.365777000$

$-0.147714000$

0.880171000

$-0.986371000$

0.356023000

$-1.092220000$

0.495584000

$-1.341884000$

$-0.712848000$

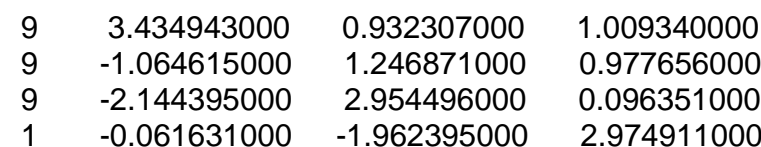

$\mathrm{CHF}_{2} \mathrm{CH}_{2}-\mathrm{SC}^{\circ}(\mathrm{OMe}) \mathrm{S}-\mathrm{CH}_{2} \mathrm{CHF}_{2}$

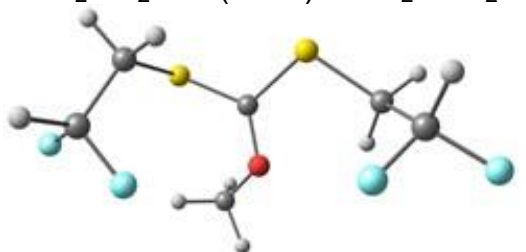$$
6
$$

.696617000

$\begin{array}{ll}16 & -3.143813000 \\ 6 & -1.187168000\end{array}$

60.079684000

$8-0.220311000$

$6 \quad 0.636694000$

$1 \quad 0.709068000$

$1 \quad 1.634867000$

$9-2.233321000$

$9-4.320676000$

$1-2.519100000$

$\begin{array}{ll}1 & -2.519100000 \\ 1 & -3.489349000\end{array}$

$16 \quad 1.694564000$

$6 \quad 2.239175000$

12.994139000

$1 \quad 1.386441000$

$6 \quad 2.837494000$

$9 \quad 1.910008000$

$9 \quad 3.882745000$

13.205925000

$1-3.311361000$

$1 \quad 0.168999000$

0.710198000

$-0.497003000$

1.493032000

0.888703000

$-0.242923000$

$-0.583106000$

0.248569000

$-0.850906000$

$-1.500688000$

$-0.949801000$

0.406725000

1.465159000

1.422080000

0.432939000

1.046653000

0.303176000

$-0.912106000$

$-1.713631000$

$-0.769269000$

$-1.436984000$

$-0.270593000$

$-1.447448000$

0.167481000

$-0.634701000$

$-0.487674000$

0.582633000

1.263609000

2.355158000

3.067364000

1.995775000

$-0.551098000$

$-0.102314000$

1.203155000

0.144539000

0.210488000

$-1.265544000$

$-1.770035000$

$-1.940048000$

$-0.925731000$

$-0.325398000$

$-0.061656000$

$-1.821205000$

$-1.697600000$

2.832577000

\section{References:}

(1) Guerre, M.; Campagne, B.; Gimello, O.; Parra, K.; Ameduri, B.; Ladmiral, V. Macromolecules 2015, 48, 7810-7822.

(2) O’Brien, J. L.; Gornick, F. J. Am. Chem. Soc. 1955, 77, 4757. 